\title{
Practicing Surgical Pathology at a Distance, Need of the Hour: Our Experience of a Single Tertiary Care Referral Cancer Centre Observational Study from Western India During Covid-19 Pandemic
}

\author{
Anjali Sharma ${ }^{1}$. Praveena G. Vyas ${ }^{1}$
}

Received: 26 June 2020 / Accepted: 9 August 2021 /Published online: 16 August 2021

(C) Indian Association of Surgical Oncology 2021

\begin{abstract}
COVID 19 pandemic struck the globe at a lightning speed mandating the use of containment measures like social distancing and work from home policies to prevent the transmission of this potentially lethal respiratory virus. Our institute, a tertiary referral cancer center catering to the whole of India (Western India in particular), implemented the "work from home" policy during COVID 19 pandemic. For the first time, the concept of "Practicing Pathology from the Distance" (telepathology) was implemented in the Department Of Pathology. This paper discusses how telepathology was factualized, integrated the problems faced during its reporting and possible solutions into the daily surgical pathology reporting at our institute. We analyzed 135 cases by Static Imagery Telepathology out of the total 385 cases reported during national lockdown in India (23rd March to 23rd May 2020) with later confirmation by light microscopy to search for percentage of diagnostic concordance and discrepancy, if any. We experienced $100 \%$ diagnostic concordance in all the cases which tested the experience skill and expertise of the concerned telepathology team. However some diagnostic challenges and technical pitfalls were noted while using static imagery technique like time constraints and image qualities. These problems could be resolved by integrating whole slide imaging telepathology for future use. We conclude that during the COVID 19 pandemic, practicing pathology at a distance by integration of technology, expertise, and team work is the need of the hour and the ultimate solution.
\end{abstract}

Keywords Telepathology $\cdot$ COVID-19 $\cdot$ Pandemic

\section{Article}

The COVID 19 which struck the entire globe at a lightning speed has geared up the world to take measures like lockdowns and curfews to curb the spread of the virus via person to person and respiratory routes. Social distancing became the norm of the day, and the concept of work from home was applied to all the work sectors. We also implemented the policy of functioning with the least possible staff on ground and the rest were advised to work from home. Pathology laboratory personnel were not exempt from this protocol. For the first time, the concept of "practicing pathology from a distance" was implemented at our hospital, a tertiary cancer referral center for the patients from all over India and

Praveena G. Vyas

prvnvyas22@gmail.com

1 Department of Pathology, Bhagwan Mahaveer Cancer Hospital and Research Center, Jaipur 302018, India
Western India in particular [1,2]. This paper discusses how telepathology was factualized and integrated the problems faced during its reporting and possible solutions for the same into the daily surgical pathology reporting at our institute.

\section{Work arrangement during lockdown period (23rd March 2020 to 23rd May 2020)}

Amidst the nationwide lockdown announced on 23rd March 2020 in India, in view of the spreading COVID 19 pandemic, the laboratory staff was divided into 2 teams working on the alternate day basis. The senior consultant (Professor Emeritus) is 80 years old and according to the national guidelines was to stay at home considering the risk of infection in senior citizens. The other Senior Consultant Histopathologist and Cytopathologist (Head of the Department) was to coordinate the everyday hospital activities with the on-duty residents and the technical staff team wise. The histopathology and cytology cases were seen on light microscopy by the senior consultant on the day of the attending the hospital. 
On other days, the on duty resident doctor (post MD 3-year experience) coordinated with the senior consultant via telepathology. Thus, a comprehensive workflow plan was implemented with support and coordination of the hospital administrators and operating oncosurgeons.

\section{Applications of telepathology at the institute}

- Reporting of frozen sections

- Reporting of cytology slides

- Reporting of small biopsies

- Reporting of big biopsies

- Reporting of ancillary investigations like immunohistochemistry (IHC) and special stains

- Review blocks and slides obtained from other hospitals for expert opinion [3]

\section{Work algorithm for telepathology at the institute}

- Specimens were accepted and kept in the primary containment area along with the requisition forms containing complete clinical history and COVID 19 negative status of the patients.

- The containers were disinfected with $0.1 \%$ sodium hypochlorite before entering the processing area.

- The resident doctor/technician on duty processed the specimens according to the request for the testing while using personal protective equipment.

- For frozen sections, the specimens were processed immediately within the TAT (turnaround time). [4]

- For fluid cytology, fluids were received in air tight leak proof containers and were processed in the biosafety cabinets.

- For small biopsies, the specimens were fixed in $10 \%$ neutral buffered formalin and then taken for further processing.

- The frozen sections were stained with rapid H\&E (Hematoxylin and Eosin) stains and given to the on duty senior resident who was well versed with the use of telepathology.

- The static telepathology was used for further reporting.

- Images from the most representative areas of the slides were captured on the calibrated Pentahead Microscope NIKON Y-THPL (JAPAN) $(9 \mathrm{~V}, 0.5 \mathrm{~A})$ at $4 \mathrm{X}, 10 \mathrm{X}$, and $40 \mathrm{X}$ resolutions. The images ranged from 3 to 15 in number per case, ranging in 2 to $4 \mathrm{MB}$ in size [5]. The Pentahead had connection to the SAMSUNG plasma TV for image viewing and finalizing the best quality images (Figs. 1 and 2). On request of the reporting consultant histopathologist, additional images were taken for further evaluation and curricular purposes.

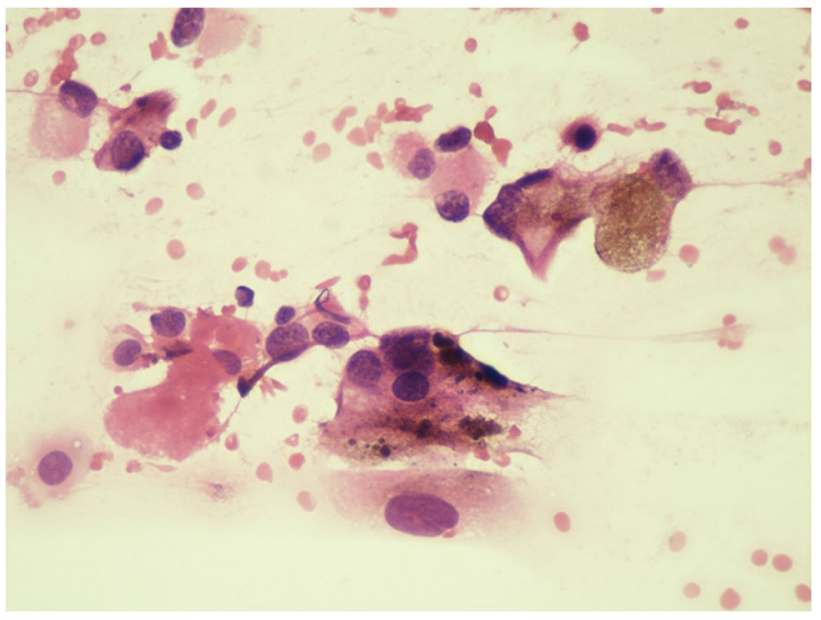

Fig. 1 Static telepathology image showing metastatic malignant melanoma in a lymph node (40X H\&E stain)

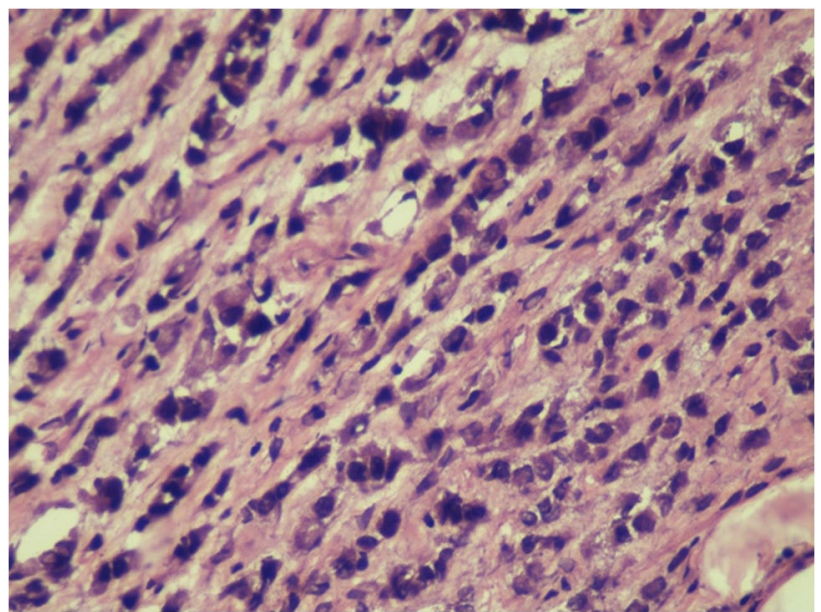

Fig. 2 Static telepathology image showing of lobular carcinoma breast (40X H\&E stain)

- A folder containing all these images and the PDF of the requisition form of each patient was prepared and saved in the desktop.

- These folder contents were emailed or sent via WhatsApp to the consultant via the Internet using a hospital VPN Internet connection comprising of 2 circuits and 2 lease lines of $20 \mathrm{Mbps}$ and $10 \mathrm{Mbps}$, each circuit working in $1: 1$ ratio.

- The consultant received the images on personal computer at home and, after careful perusal, gave the diagnosis as a reply to the previously sent email and also as an extra measure telephonically informed to the on duty senior resident.

- The frozen section final report was then informed to the concerned surgeon telephonically by the Consult- 
ant Pathologist from home. A written note regarding the same was made on the requisition form by the resident.

- The report on the requisition form was written by the on duty senior resident and handed over to the transcriptionist.

- The transcriptionist also received the e mail containing the specific requisition form and the diagnosis from the consultant for the purpose of digitalizing the report and dispatching it online within the turn-around time. The reports of telepathology were entered in the LIS (Laboratory Information System) and the LIS was accessed remotely, so that TAT could be met.

- All the cytology slides and the CSF (cerebrospinal fluid) cytology slides were mandatorily screened and sent for reporting by telepathology for rapid reporting. The consultant reported the final impression for the cytology slide imagery via email to the on duty resident and the transcriptionist for digitalizing and releasing of the report the same day. So, this way the TAT was maintained in all cases.

- The small biopsies were also screened, and necessary instructions regarding the ancillary investigations like special stains and immunohistochemistry (IHC) were given to the concerned technicians. The final reporting was done the next day by the consultant, and reports were signed in person and released. In cases requiring ancillary testing, TAT could be maintained because of telepathology, which helped in timely selection and ordering ancillary tests.

- For big biopsies, small biopsies, review slides, those which had a dubious diagnosis, and those requiring the ancillary tests like special stains and IHC were sent via email and WhatsApp to the consultant for further advice and opinions. The necessary panels were advised and replied back via emails and telephonic conversations. All these cases were perused by the consultant the next day and reported and signed the cases in person.

Therefore, stringent maintenance of the turnaround time for frozen sections (30 min), CSF cytology (1 day), fine needle aspiration and fluid cytology (3 days), small biopsies ( 3 days), and big biopsies ( 7 days) was ensured at all levels.

- We compiled the total number of cases reported during the lockdown period (23rd March 2020 to 23rd May 2020) and stratified them into those reported by only light microscopy and those reported by telepathology. Out of the total 385 cases reported, 135 cases were reported static telepathology on the days of non-availability of the consultant in the department due to social distancing protocol and time table schedule designed for the COVID situation. These 135 cases were next day confirmed by light microscopy and thus the diagnostic concordance was studied for them. Special attention was given for dis-
Table 1 Organ systemwise distribution of cases reported by telepathology

\begin{tabular}{ll}
\hline Organ system & $\begin{array}{l}\text { Number of cases } \\
\text { reported by static } \\
\text { pathology }\end{array}$ \\
\hline Head neck & 37 \\
Central nervous system & 04 \\
Lymph nodes & 10 \\
Lung & 06 \\
Breast & 35 \\
Soft tissue and bones & 11 \\
Gastrointestinal tract & 12 \\
Female genital tract & 16 \\
Male genital tract & 04 \\
\hline
\end{tabular}

crepancy, if any; the plausible reason for the same was searched. The rest of the cases were reported in person by the consultant in the department on alternate day basis. Table 1 shows organ systemwise distribution of cases reported by static telepathology.

Prior to the COVID era, the static telepathology was never used for routine reporting in the department. Hence it was not possible to compare the diagnostic concordance of static telepathology reporting during non-COVID times.

One hundred percent concordance in the diagnosis was established due to the experience, skill, and expertise at both the sender's and the receiver's end. Similar results $(95$ - $100 \%$ concordance between glass slides and telepathology) were also observed by two different Indian studies by Baruah M. K. and Feroz et al., respectively [6, 7]. Table 2 shows comparison and diagnostic concordance between light microscopy and static telepathology.

The resident doctor was well versed with the finesse and the nuances of static telepathology and could successfully target the diagnostic areas from the slide for further perusal of the consultant histopathologist. Whenever in doubt or uncertainty about the key areas, additional images were requested by the reporting consultant which were deftly forwarded and also avidly discussed by both on telephone. The clinical history was telephonically provided by the concerned surgeon. Static telepathology was our only option till validation of the whole slide imaging telepathology at our institute. The Head of the Department (consultant histopathologist) has already undertaken a validation based research project of 5000 histopathology slides to be reported by whole slide imaging technique after ethical approval.

\section{Issues faced during telepathology [7-9]}

- Poor Internet connectivity. 
Table 2 Comparison and diagnostic concordance between light microscopy and telepathology

\begin{tabular}{|c|c|c|c|c|c|}
\hline & Total cases & $\begin{array}{l}\text { Average TAT for static telepathology } \\
\text { and followed by light microscopy }\end{array}$ & $\begin{array}{l}\text { Cases reported by static telepathology } \\
\text { and followed by light microscopy }\end{array}$ & $\begin{array}{l}\text { Diagnostic } \\
\text { concordance }\end{array}$ & Discrepancy \\
\hline Cytology & 92 & 3 days & 35 & $100 \%$ & None \\
\hline Frozen section & 60 & $30 \min$ & 09 & $100 \%$ & None \\
\hline Small biopsy & 99 & 3 days & 45 & $100 \%$ & None \\
\hline Big biopsy & 58 & 4 days & 25 & $100 \%$ & None \\
\hline Review slides & 26 & 3 days & 11 & $100 \%$ & None \\
\hline $\mathrm{IHC}$ & 50 & 2 days & 10 & $100 \%$ & None \\
\hline Total & 385 & & 135 & $100 \%$ & None \\
\hline
\end{tabular}

- Limitations of static imagery as compared with the whole slide imaging technique. This shortcoming can be overcome by preferring whole slide imaging technique and involving both teams (senders and receivers) in active participation in the slide evaluation and more freedom of visualization of the areas of interest in the slides.

- Image qualities in terms of light adaptation, blurring, and resolutions.

- Difficulty in selecting the most representative areas from the slide.

- Time required in capturing all the relevant images.

- Pressure to maintain TAT for frozen sections.

\section{Diagnostic challenges}

One can infer from all the discussed facts that telepathology proved a wonderful solution for histopathology reporting during COVID 19 pandemic, but some cases that were encountered during these testing times definitely proved to be diagnostically challenging. One such case was that of a 1-year-old infant whose abdominal mass biopsy caused a diagnostic dilemma between labelling the neoplasm as neuroblastoma or nephroblastoma, and thus our diagnosis was that of a round cell neoplasm. Sometimes the difficulty and confusion also arose in differentiating between the verrucous carcinoma and well-differentiated squamous cell carcinoma in the oral cavity punch biopsies. The fine needle aspiration cytology smears and cell blocks from the suspicious breast lumps proved to be challenging especially in differentiating between the atypical ductal hyperplasia and lowgrade DCIS and also for some papillary lesions. Some ascitic and pleural fluid cytology smears proved tricky as it was occasionally difficult to label the mesothelial cells as reactive or atypical. All these dilemmas led to much discussion and pondering by the diagnostic team and finally an honest report regarding the dilemmas were reported to the treating surgeons who agreed for the final confirmation of such cases by detailed routine processing and ancillary tests like immunohistochemistry. Only these seven cases were deferred for the light microscopy review. The limitations faced due to static pathology images were eventually resolved on light microscopy and ancillary testing.

\section{Conclusions}

As it is rightly said that necessity is the mother of invention, so has this COVID 19 pandemic taught us to adapt to the changing scenario by incorporating technology with expertise and utilizing telepathology as the new mode of pathology reporting [1]. Static telepathology became the neonorm mode of reporting during nationwide lockdown keeping the department functional and ensuring the safety of the staff on duty. The static imagery mode of reporting expressed satisfactory concordance and adherence to the maintenance of TAT. We conclude that during the COVID 19 pandemic, practicing pathology at a distance by integration of technology, expertise, and team work is the need of the hour and the ultimate solution.

Authors' Contributions Not applicable.

Data Availability Yes.

Code Availability Not applicable.

\section{Declarations}

Ethics Approval Not applicable.

Consent to Participate Not applicable.

Consent for Publication Not applicable.

Conflict of Interest There are no conflicts/competing of interests. 


\section{References}

1. Meyer J, Paré G (2015) Telepathology impacts and implementation challenges: a scoping review. Arch Pathol Lab Med 139(12):1550-1557. https://doi.org/10.5858/arpa. 2014-0606-RA

2. Orah N, Rotimi O (2019) Telepathology in low resource African settings. Front Public Health 7:264. https://doi.org/10.3389/fpubh. 2019.00264

3. Sankaye S, Kachewar, S (2011) Telepathology for effective healthcare in Developing Nations. Australas Med J. 592-5. https://doi. org/10.4066/AMJ.2011.855.

4. Huang Y, Lei Y, Wang Q, Li D, Ma L, Guo L et al (2018) Telepathology consultation for frozen section diagnosis in China. Diagn Pathol. 13(1):29. Available from:https://doi.org/10.1186/ s13000-018-0705-0

5. Baruah MK, Jayaram N, Prasad SR, Bedi S, Johnson JM, La Rosa FG (2001) Telepathology in India. Indian J Pathol Microbiol 44(3):381-383
6. Baruah MK (2005) The practice of telepathology in India. J Postgrad Med 51(4):316-318

7. Feroz A, Mohammed Feroz TP, Bastian TS, Selvamani M (2020) Telepathology: an update on applications, latest advances, and current status in Indian scenario. J Cancer Res Ther 16(4):703707. https://doi.org/10.4103/jcrt.JCRT_477_17

8. Farahani N, Pantanowitz L (2015) Overview of telepathology. Surg Pathol Clin 8(2):223-231. https://doi.org/10.1016/j.path. 2015.02.018

9. Farahani N, Riben M, Evans AJ, Pantanowitz L (2016) International telepathology: promises and pitfalls. Pathobiology 83(23):121-126. https://doi.org/10.1016/j.path.2015.02.018

Publisher's Note Springer Nature remains neutral with regard to jurisdictional claims in published maps and institutional affiliations. 